\title{
Aprendizaje basado en problemas (ABP) como estrategia para adquisición de competencias genéricas: estudiantes de nutrición y dietética, Universidad de La Frontera
}

\author{
Problem-based learning as a strategy \\ for acquisition of generic skills: \\ students of nutrition and dietetics, \\ Universidad de La Frontera
}

\begin{abstract}
The study of generic skills in the training of health professionals has become an instrument of increasing value to the institutions of higher education and health centers. In order to know in detail the opinions of students of the career of nutrition and dietetics of the Faculty of Medicine, Universidad de La Frontera, Temuco-Chile related to the generic competences acquired during their training process, 5th year students took part in two focus groups, after signing an informed consent. The analysis of data followed a scheme of progressive reduction of them, validating by triangulation of researchers. The results represent the consistency that must exist between study and educational plan didactics in the development of the generic competences, so necessary nowadays for the work insertion of health professionals.

Key words: Professional competence; nutritionist; human resources formation; professional education, students, health occupations.
\end{abstract}

Eugenia Saavedra R. (1) Mónica Illesca P. (1) Mirtha Cabezas G. (2)

(1) Departamento de Medicina Interna, Facultad de Medicina, Universidad de La Frontera, Temuco, Chile. (2) Departamento Ciencias Preclínicas, Facultad de Medicina, Universidad de La Frontera, Temuco, Chile.

Dirigir la correspondencia a: Profesora Eugenia Saavedra Romero Departamento de Medicina Interna. Facultad de Medicina, Universidad de La Frontera Manuel Montt 112, Temuco E-mail: eugenia.saavedra@ufrontera.cl

Este trabajo fue recibido el 15 de Julio de 2013 y aceptado para ser publicado el 1 de Abril de 2014.

\section{INTRODUCCIÓN}

Las Universidades, producto de la globalización, han debido modificar su sistema educativo con el propósito de formar individuos con los saberes, habilidades, actitudes y valores requeridos para enfrentar un nuevo ámbito laboral (1, $2)$, requiriendo profesionales competentes con conocimientos profundos de su realidad y para asumir un compromiso ético con la sociedad.

El concepto de competencia a finales de la década de los 60 en Columbia Británica y Canadá fue resultado de la estructuración de un currículo en el que se pudiera evaluar el dominio de un comportamiento determinado, con un instrumento objetivo. A su vez, a principios de los 80 , los países industrializados observan un profundo desfase entre los perfiles de egreso, muy especializados, con los requerimientos del mercado que exigía características más dinámicas y flexibles en los trabajadores $(3,4)$.

Existen diversas definiciones para este término: "pericia, aptitud, idoneidad para hacer algo o intervenir en un asunto determinado" (5); combinación de conocimientos, capacidades y comportamientos que se pueden utilizar e implementar directamente en un contexto de desempeño, siendo las nociones de "combinación" y de "contexto" esenciales (6); capacidad de generar aplicaciones o soluciones adaptadas a la situación, movilizando los propios recursos y regulando el proceso hasta lograr la meta pretendida (7); complejas capacidades integradas, en diversos grados, que la educación debe formar en los individuos para que puedan desempeñarse como sujetos responsables en diferentes situaciones y contextos de la vida social y personal, sabiendo ver, hacer, actuar y disfrutar convenientemente, evaluando alternativas, eligiendo las estrategias adecuadas y haciéndose cargo de las decisiones tomadas (8). De todas ellas se rescata la relevancia que se le atribuye tanto a los atributos individuales como a la caracterización de funciones y tareas en las que se pondrán en acción esas cualidades y la serie de condiciones para su realización.

Por otra parte, la clasificación es diversa en nomenclaturas y taxonomías (9); independiente de los autores, en este estudio se considera lo establecido por el Informe Tuning, las que se catalogan en específicas y genéricas. Las primeras, correspon- 
den a cada profesión sin constituirse en un desafío mayor para los centros formadores, en tanto las segundas comprenden a aquellas que son comunes a cualquier profesión y que en estos momentos son requeridas por los empleadores.

Las competencias genéricas se subdividen en instrumentales (que puede ser a su vez cognoscitiva, metodológica, tecnológica y lingüística), sistémicas (destrezas y habilidades que conciernen a los sistemas como totalidad, suponen una combinación de comprensión, sensibilidad y conocimiento que permiten al individuo ver cómo las partes de un todo se relacionan y se agrupan) e interpersonales (facilitan los procesos de interacción social, cooperación y comunicación, expresión de compromiso social o ético) (8)

En este contexto, para las Instituciones de Educación Superior, que ofrecen carreras del área de la salud, el estudio de las competencias en el proceso de formación de los futuros profesionales, se ha convertido en un instrumento de valor creciente, ya sea para investigar su adquisición, desarrollo y fortalecimiento, como también la repercusión de éstas en el desempeño laboral. Más aún, las exigencias del entorno, ya sea Ministerio de Salud, de Educación, empleadores o comunidad, obligan a las Universidades a implementar estrategias metodológicas pertinentes para estos fines.

Una de ellas es el aprendizaje basado en problemas (ABP), como lo demuestra un estudio que incorporó la estrategia a las asignaturas de Microbiología y Parasitología, validando con ello la importancia de planificar y disponer de un modelo de enseñanza con múltiples técnicas educativas, que considere los estilos de aprendizaje de los educandos, para favorecer el desarrollo de competencias genéricas y específicas propuestas en el perfil de egreso (10). Otra investigación demostró que las personas formadas con ABP expresaron tener mayor seguridad para enfrentar la práctica basada en la evidencia y un acentuado pensamiento crítico a diferencia de los sin ABP (11). A su vez, el trabajo de ABP con mapas conceptuales aumentan las habilidades de pensamiento crítico y disposición personal al aprendizaje autodirigido con mejora en la capacidad de estudio independiente, razonamiento, interacción con grupos y participación activa (12). La comunicación, el autoaprendizaje e iniciativa personal, flexibilidad, formación ciudadana y sensibilidad social son otras competencias genéricas identificadas con esta didáctica educativa (13).

En este sentido, la Carrera de Nutrición y Dietética de la Universidad de La Frontera, desde el año 2003, dio inicio al nuevo Plan de Estudio, enmarcado en el constructivismo, centrado en el estudiante, basado en los principios de educación del adulto y con estrategia metodológica del ABP, entre otras, con el propósito de propender a la formación de un titulado con un perfil que además de las competencias específicas desarrollen las genéricas.

Sin embargo, a través de la observación empírica y conversaciones informales con los estudiantes de quinto año, se desprende que algunas de ellas estipuladas por la Carrera no se visualizan en su totalidad (toma de decisiones de acuerdo a nivel curricular, liderazgo, trabajo en equipo, autoaprendizaje, entre otras), aunque hacen referencia de haber cursado actividades curriculares integradas, específicamente módulos multiprofesionales durante los dos primeros años donde sí identifican haber trabajado en equipo, desarrollar capacidad de análisis, crítica y autocrítica y liderazgo.

Con el propósito de orientar las actuaciones pedagógicas y organizativas destinadas a mejorar la calidad y la gestión de la docencia, se realiza esta investigación con el objetivo de conocer la opinión de los estudiantes de quinto año de la
Carrera de Nutrición y Dietética de la Facultad de Medicina de la Universidad de la Frontera, con relación a las competencias genéricas adquiridas durante su proceso de formación en el segundo semestre del 2010. Los específicos se enfocan en a) indagar el concepto "competencia genérica", b) consultar respecto a las metodologías educativas que contribuyen al desarrollo de competencias genéricas, c) explorar sobre las competencias genéricas desarrolladas con el ABP y d) develar factores que interfieren en su desarrollo.

La preeminencia de ejecutarla radica en que el conocimiento y actitud del profesorado y alumnado hacia las tareas implícitas en la formación universitaria, puede representar uno de los puntos claves de todo el progreso de la mejora docente, lo que contribuye a certificar el desempeño de los futuros profesionales requeridos por los empleadores, en este momento (14).

\section{MATERIAL Y MÉTODO}

Considerando la naturaleza del objeto de investigación, se realizó un estudio cualitativo exploratorio, descriptivo e interpretativo. Se identificó el estudio intrínseco de caso, ya que permite profundizar la comprensión y especificidades, centrándose el interés en el caso particular (15), específicamente, la Carrera de Nutrición y Dietética de la Universidad de La Frontera.

La muestra seleccionada fue intencionada de casos por criterios, tipo no probabilístico. Como en este tipo de investigación no interesa la representatividad, sino lo fundamental es conocer las vivencias de los involucrados respecto a lo que se estudia (16); se conformó un total de 16 sujetos, cuyos criterios de inclusión fueron: estar en la Práctica Profesional Controlada durante el segundo semestre 2010, tener disponibilidad horaria y firmar el Consentimiento Informado.

Para la recolección de datos, se realizaron dos grupos focales, de ocho integrantes cada uno, considerando la participación de tres personas: una para registrar las notas de campo, otra para grabar la información y la investigadora principal quién guió la discusión la cual fue libre y espontánea en relación a los temas considerados interesantes para la investigación.

Los datos se recopilaron hasta llegar al punto de saturación, es decir, reunir pruebas y evidencias suficientes para garantizar la credibilidad de la investigación (17). Para el análisis se utilizó el método de comparación constante, es decir, no se agruparon en categorías predeterminadas, sino más bien emergieron de un proceso de razonamiento inductivo, a través de un método generativo y constructivo en el que se combinó la codificación inductiva de categorías con la comparación constante de ellas (18). Se adoptó el esquema de reducción progresiva (separación de unidades, agrupamiento, identificación y clasificación de elementos), disposición, transformación y obtención de conclusiones verificables (17). Como herramienta computacional se utilizó el programa ATLAS - ti. Cabe mencionar que este proceso sistemático, ordenado y flexible fue concurrente con la recogida de datos. El nivel progresivo de reducción y estructuración teórica fue a través de tres niveles:

Nivel 1: segmentación e identificación de unidades de significado (codificación) y agrupación en categorías descriptivas de los fragmentos de texto con sentido semántico relacionado directamente con el carácter reflexivo desde el punto de vista del informante.

Nivel 2: construcción de un sistema de núcleos temáticos emergentes o metacategorías (dimensión interpretativa del investigador, a partir de los conceptos del primer nivel).

Nivel 3: identificación de dominios cualitativos (análisis 
secuencial y transversal de las metacategorías).

La rigurosidad científica se garantizó utilizando los criterios de rigor determinado por las estrategias de veracidad: credibilidad, transferibilidad, dependencia y confirmabilidad (19). Cabe señalar que para la credibilidad se realizó la triangulación por investigador, experto, registro y documentación de toda la información obtenida.

\section{RESULTADOS Y DISCUSIÓN}

Nivel 1: una vez identificadas todas las unidades de significado que hacían alusión a una misma idea se seleccionaron las que eran relevantes para los objetivos del estudio descartando aquéllas que no tenía ningún tipo de relación. Las categorías se codificaron con tres y cuatro letras que, en general coincidían con las primeras de la idea a la que la categoría se refería. Al final de este proceso se develaron 174 unidades de significado relevantes para el estudio agrupado en 5 categorías emergentes. La de mayor y menor frecuencia fueron la de los "Factores que interfieren en el desarrollo de las competencias genéricas" (46) y los "Factores que favorecen el desarrollo de competencias genéricas" (20). A su vez la identificación de "competencias genéricas" fue ampliamente reconocida (44). En tanto las "Competencias genéricas desarrolladas con la metodología educativa" fue mencionada 38 veces y las "Metodologías educativas que contribuyen al desarrollo de competencias genéricas" en 26 oportunidades (tabla 1). La definición de

\section{TABLA 1}

Distribución de frecuencias de las unidades de significado encontradas en los discursos.

\begin{tabular}{|c|c|c|}
\hline Código & Categoría & $\begin{array}{c}\text { Frecuencia Unidades } \\
\text { de Significado }\end{array}$ \\
\hline FIDC & Factores que interfieren en el desarrollo de las competencias genéricas: & 46 \\
\hline CGEN & Competencias genéricas & 44 \\
\hline$C D M$ & Competencias genéricas desarrolladas con la metodología educativa & 38 \\
\hline MDC & Metodologías educativas que contribuye al desarrollo de competencias genéricas & 26 \\
\hline \multirow[t]{2}{*}{ FFDC } & Factores que favorecen el desarrollo de competencias genéricas & 20 \\
\hline & & 174 \\
\hline
\end{tabular}

TABLA 2

Distribución de frecuencias de las unidades de significado de "categoría factores que interfieren en desarrollo de las competencias genéricas" (FIDC).

Código

EST Estudiantes

ECU Estructura curricular

RDE Relación docente-estudiantes

RAA Recursos y apoyo para el aprendizaje

\section{Categoría FIDC}

Personalidad

Edad

Competitividad entre estudiantes

No solidaridad

Lugar de residencia

No actualización docentes

Nivel de inicio de la malla

Resistencia de docentes

Actualización en malla curricular

Desorganización

Centro de práctica

Distancia profesor-estudiante

Miedo al profesor

Relación con preceptor

Respeto al profesor

Recursos bibliográficos

Internet
Frecuencia unidades de significado $\mathrm{N}^{\circ} \quad \%$

10

3

6.5

3

6.5

1

2.2

1

2.2

5

10.8

4

8.7

3

6.5

1

2.2

1

2.2

1

2.2

$6 \quad 13.0$

$3 \quad 6.5$

$1 \quad 2.2$

$1 \quad 2.2$

$1 \quad 2.2$

$1 \quad 2.2$

46 
competencia, no emergió, la cual estaba formulada como uno de los objetivos específicos del estudio.

Se puede destacar en este estudio que el desarrollo de las competencias se ve afectado por: "estudiantes" (39.1\%), "estructura curricular" (32.6\%), "relación docente-estudiantes" (23.9\%) y "recursos y apoyo para el aprendizaje" (4.4\%). El factor dependiente de los educandos, tales como edad, personalidad, falta de solidaridad, competitividad entre ellos y lugar de residencia, son los que perciben como obstaculizadores para el logro de ellas. En esta categoría resalta la capacidad de autocrítica de los estudiantes dado que las otras tienen un menor porcentaje (tabla 2).

Con un $90 \%$ de las menciones fueron identificadas las unidades de significado que conformaron la "categoría factores que favorecen el desarrollo de las competencias genéricas" (tabla 3). Aquí aparecen la motivación y confianza al igual que la estructura curricular como factores que facilitan la generación de dichos atributos.

En la distribución de frecuencia de las unidades de significado de "categoría competencias genéricas" (tabla 4), si bien es cierto los estudiantes hablan de liderazgo, proactividad, compañerismo, entre otras, ninguna es reconocida como

\section{TABLA 3}

Distribución de frecuencias de las unidades de significado de "categoría factores que favorecen el desarrollo de las competencias genéricas" (FFDC).

\begin{tabular}{|c|c|c|c|}
\hline \multirow[t]{4}{*}{ Código } & \multirow[t]{2}{*}{ Categoría FFDC } & \multicolumn{2}{|c|}{$\begin{array}{c}\text { Frecuencia unidades } \\
\text { de significado }\end{array}$} \\
\hline & & $\mathrm{N}^{\circ}$ & $\%$ \\
\hline & Evaluación continua de competencias genéricas & 3 & 15.0 \\
\hline & Práctica clínica & 3 & 15.0 \\
\hline \multirow[t]{4}{*}{ ECU Estructura curricular } & Carrera motivación curso extras & 1 & 5.0 \\
\hline & Semana de la Carrera & 1 & 5.0 \\
\hline & Padrinos & 1 & 5.0 \\
\hline & Rol del tutor & 1 & 5.0 \\
\hline \multirow[t]{3}{*}{ EST Estudiantes } & Confianza & 5 & 25.0 \\
\hline & Motivación & 2 & 10.0 \\
\hline & Valoración de sí mismo (autoconcepto) & 1 & 5.0 \\
\hline \multirow[t]{3}{*}{ INS Institucional } & Talleres de la Universidad & 1 & 5.0 \\
\hline & Todo el entorno & 1 & 5.0 \\
\hline & & 20 & 100 \\
\hline
\end{tabular}

TABLA 4

Distribución de frecuencias de las unidades de significado de "categoría competencias genéricas" (CGEN).

Código

Categoría CGEN

INT Interpersonal

SIS Sistémicas

INTR Instrumentales
Liderazgo

Trabajo en equipo

Equipo multiprofesional

Proactividad

Compañerismo

Responsabilidad

Aprender a aprender, autoaprendizaje,

Análisis crítico, pensamiento crítico

Autocrítica

Idioma (inglés)

Comunicación efectiva
Frecuencia unidades de significado $\mathrm{N}$ $\%$ 11 25.0

8 18.2 4 9.1 
competencia genérica. Los relatos son coincidentes con los de la Institución. Las interpersonales con un 65.9\%, destacándose el "liderazgo" y "trabajo en equipo", atributos probablemente adquiridos desde el primer año en los módulos multiprofesionales. En las sistémicas, con un $20.5 \%$, se puede concluir que "aprender a aprender", "autoaprendizaje", "análisis crítico", "pensamiento crítico" representan características obtenidas durante el trabajo en grupo pequeño con aprendizaje basado en problemas. De las instrumentales, llama la atención que la "comunicación efectiva" logró un 4.5\%, hecho que en un profesional del área de la salud debiera tener predominancia en la formación. El idioma Inglés con un $9.1 \%$ se debe a la política Universitaria que incluye en los Planes de Estudio hasta el cuarto año la exigencia de un nivel intermedio.

En cuanto a la "categoría metodologías educativas que contribuyen al desarrollo de las competencias genéricas" (tabla 5), los estudiantes prefieren las que los convierten en protagonistas del proceso, como las tutorías o trabajo en grupo pequeño con $\mathrm{ABP}$ (donde aprenden a través de un caso clínico), junto con los debates y la implementación del portafolio (46.3\%). Las actividades prácticas y teórico -práctico como las informales (huelgas estudiantiles) son maneras efectivas donde suelen expresarse estos atributos positivos de la personalidad ( $38.5 \%$ y $15.4 \%$ respectivamente). Aquí se confirma lo encontrado en la literatura que los dominios del saber ser y del saber estar se manifiestan en la continua interacción con el otro. Aquellas clasificadas como "teórica-práctica" son valoradas en baja proporción, pudiéndose interpretar como que en estas metodologías la participación $y$, por ende, la responsabilidad individual tendría un menor peso o incidencia en la formación general.

En la "categoría competencias genéricas desarrolladas con la metodología educativa" (tabla 6), con un $71 \%$, son reconocidas las interpersonales: "trabajo en equipo" y "organi-

TABLA 5

Distribución de frecuencias de las unidades de significado de "categoría metodologías educativas que contribuye al desarrollo de competencias genéricas" (MDC).

\begin{tabular}{|c|c|c|c|}
\hline \multirow[t]{3}{*}{ Código } & \multirow[t]{2}{*}{ Categoría MDC } & \multicolumn{2}{|c|}{$\begin{array}{c}\text { Frecuencia unidades } \\
\text { de significado }\end{array}$} \\
\hline & & $\mathrm{N}^{\circ}$ & $\%$ \\
\hline & Tutorías con ABP & 7 & 27 \\
\hline \multirow[t]{3}{*}{ TEO teoría } & Debates & 3 & 11.6 \\
\hline & Portafolio & 2 & 7.7 \\
\hline & Prácticas clínicas & 4 & 15.4 \\
\hline \multirow[t]{3}{*}{ PARA práctica } & Clínica móvil (paro estudiantil) & 4 & 15.4 \\
\hline & Presentaciones en centros & 2 & 7.7 \\
\hline & Seminarios & 1 & 3.8 \\
\hline \multirow[t]{4}{*}{ TEPR teoría - práctica } & Foros & 1 & 3.8 \\
\hline & Trabajo de grupo & 1 & 3.8 \\
\hline & Talleres & 1 & 3.8 \\
\hline & & 26 & 100 \\
\hline
\end{tabular}

TABLA 6

Distribución de frecuencias de las unidades de significado de "categoría competencias genéricas desarrolladas con la metodología educativa" (CDM).

Código

INT Interpersona

SIS Sistémicas

INTR Instrumentales
Categoría CDM

Trabajo en equipo, organización Liderazgo, toma decisión

Responsabilidad social, responsabilidad

Proactivo (emprendimiento)

Autoaprendizaje (aprender a aprender)

Análisis crítico

Comunicación
Frecuencia unidades de significado

$\mathrm{N}^{\circ} \quad \%$

$14 \quad 36.8$

$\begin{array}{ll}9 & 23.7\end{array}$

$4 \quad 10.5$

$3 \quad 7.9$

$3 \quad 7.9$

$2 \quad 5.3$

$3 \quad 7.9$

$38 \quad 100$ 
zación" (36.8\%), "liderazgo" y "toma de decisiones" (23.7\%), responsabilidad tanto individual como social (10.5\%). En las sistémicas (13.2\%), el "autoaprendizaje (aprender a aprender)" (7.9\%), predomina al "análisis crítico" (5.3\%). En las instrumentales sólo identifican la "comunicación" (7.9\%). Los resultados expuestos pudieran atribuirse a que los estudiantes desde el inicio del proceso de formación trabajan con ABP.

Nivel 2: el análisis de datos indica que siguiendo con el procedimiento de formar conjuntos semejantes con todos los elementos presentados por los informantes claves se redujeron a tres núcleos temáticos: "conocimiento de las competencias genéricas", "relación entre la didáctica educativa y desarrollo de competencias genéricas" y "factores asociados al desarrollado de competencias genéricas". Es dable destacar en este espacio que el primero de ellos "competencias genéricas", según los relatos de los estudiantes, el $25.3 \%$ son adquiridas en los primeros años de la Carrera, específicamente en los módulos de Gestión e Investigación en Salud, confirmando los postulados del proceso de innovación curricular en la Facultad (2003) y lo solicitado al momento de buscar un empleo.

A su vez, los factores que interfieren para su desarrollo corresponde al $26.4 \%$ (tabla 2 ). Al respecto se puede mencionar dos hechos relevantes: el primero habla de lo que ellos visualizan como impedimento, relatados por "la personalidad de los estudiantes" (1,7\%) y el segundo son los asociados a los docentes "distancia entre profesor" (13,0\%), sumado a la "no actualización docente" (10.8\%). El segundo factor es posible de mejorar en la medida que se tome conciencia de la importancia que le confieren los estudiantes a estos hechos.

Nivel 3: posterior al análisis secuencial y transversal de las metacategorías, emergen dos dominios cualitativos: "concepción de competencias genéricas del egresado de nutrición de la UFRO", semejante a los atributos clasificados según Tuning 2003 (8) y "Correspondencia entre el plan de estudio y desarrollo de competencias genéricas", que expresa las opiniones en relación a la potenciación de estas competencias con la metodología educativa empleada ya sea teórico, práctico o teórico-práctico, y los factores que favorecen e interfieren en el desarrollo de ellas.

De acuerdo a esto, el primer dominio es representativo de la pregunta de investigación, mientras que el segundo corresponde a las unidades de significado que dan cuenta de cómo se adquieren, qué interfiere y cuáles elementos les favorecen dichas cualidades en el Plan de Estudio de la Carrera.

Resulta relevante tener presente que las estrategias y metodologías centradas en el estudiante como tutorías en grupo pequeño, confección de portafolio y prácticas enriquecen el desarrollo de las competencias genéricas y que el rol docenteformador se debería fortalecer para apoyar estos aprendizajes.

\section{RESUMEN}

El estudio de competencias genéricas en la formación de profesionales de la salud se ha convertido en un instrumento de valor creciente para las instituciones de educación superior y centros asistenciales. Con el objetivo de conocer en detalle las opiniones del alumnado de la Carrera de Nutrición y Dietética, de la Facultad de Medicina de la Universidad de La Frontera, Temuco-Chile relacionadas con las competencias genéricas adquiridas durante su proceso de formación, se realizó una investigación cualitativa, mediante estudio de caso. Participaron estudiantes de $5^{\circ}$ año en dos grupos focales, previa firma de consentimiento informado. El análisis de datos siguió un esquema de reducción progresiva de ellos, validándose por triangulación de investigadores. Los resultados representan la congruencia que debe existir entre el plan de estudio y didáctica educativa en el desarrollo de competencias genéricas, tan necesarias en el mundo actual para la inserción laboral de profesionales de la salud.

Palabras clave: Competencia profesional; nutricionista; formación de recursos humanos; educación profesional; estudiantes del área de la salud.

\section{BIBLIOGRAFÍA}

1. Tedesco J. Educar en la sociedad del conocimiento, 2000. Recuperado el 09 de abril de 2013, de http:// www.nc.mictlansoft.com/pdf/Resumen $\% 20$ Libro $\% 20$ Educar\%20en\%20Soc\%20de1\%20Conocimiento.\%20 JCTedesc.pdf

2. Ospina A. Currículo por en competencias en la Universidad de la Sabana. CHÍA 2006; 6(1): 117-24.

3. Tünnermann C. La universidad latinoamericana ante los retos del siglo XXI. 2003, Colecciones UDUAL, México. Recuperado el 4 de mayo de 2013, de http://www.udual. org/CIDU/ColUDUAL/Tunner/capitulo4.pdf

4. Tobón, S. Formación basada en competencias. Pensamiento complejo, diseño curricular y didáctica. Ecoe, Bogotá, 2006.

5. Real Academia Española, 2010. Recuperado el 30 de noviembre de 2012 de http://adultosuperdotado.foroactivo.com/t390-nueva-actualizacion-del-diccionario-de-lareal-academia-espanola-drae

6. Le Boterf, G.; Vincent, F. y Barzucchetti, S. Como gestionar la calidad de la formación. Edipe, Barcelona, 1995.

7. Rey, B. Les competénces transversales en question. ESF, Paris, 1996.

8. Tuning Educational Structures in Europe Informe Final Proyecto Piloto-Fase 1. Edición y Cultura. Sócrates, Universidad de Deusto - Universidad de Groningen, 2003.

9. Solar, Ma . El currículo de competencias en la educación superior: desafíos y problemáticas. Rev Pensamiento Educativo 2005; 36, 172-91.

10. Hechenleitner M. Madrid V. Rojas P. y Ortiz L. Evaluación de una intervención didáctica de la asignatura de microbiología y parasitología según opinión de los estudiantes de la Carrera de Nutrición y Dietética. Rev Educ Ciencias Salud 2008; 5(2): 92-6.

11. Applin H. Williams B. Day R. y Buro K. A comparison of competencies between problem-based learning and nonproblem-based graduate nurses. Nurse Education Today 2011; 31: 129-34.

12. Tseng H. Chou F. Wang H. Ko H. Jian S. y Weng W. The effectiveness of problem-based learning and concept mapping among Taiwanese registered nursing students. Nurse Education Today 2011; 31: 41-6.

13. Labraña A. Durán E. Soto D. Competencias del Nutricionista en el ámbito de Atención Primaria de Salud. Rev Chil Nutr. 2005; 32(3): 239-46.

14. Kyriacou, C. Developing your teaching skills. Basil Blackwell, Oxford, 1991.

15. Stake, R. Investigación con estudio de casos. Morata, Madrid, 1999

16. Álvarez-Gayou, J, J.L. Cómo hacer Investigación Cualitativa. Fundamentos y Metodología. México: Paidos Educador, 2003.

17. Miles, M. y Huberman, A. Qualitative data análisis: a sourcebook of new methods. Stage, CA, 1984.

18. Glaser, B. y Strauss, A. The discovery of Grounded Theory. Aldine, Chicago; 1997.

19. Guba, E. y Lincoln, Y. Naturalist Inquirí. Sage, London, 1985. 